\title{
Sul problema non lineare di Stefan in strati cilindrici o sferici.
}

Memoria di Grorgro Sestini (a Firenze)

Ad Antonio Signorini nel suo $70^{\text {mo }}$ compleanno.

\begin{abstract}
Sunto. - Si estendono al caso di strati cilindrici o sferici $i$ risultati esposti in altra memoria relativi al problema unidimensionale non lineare di Stefan per lo strato piano, conseguendo un teorema di esistenza ed unicitò per le soluzioni del problema non lineare in esame.
\end{abstract}

\section{Introduzione}

In una recente memoria [3] $\left(^{*}\right)$, che nel seguito sarà richiamata con $(M)$, ho dimostrato, con procedimento classico, l'esistenza di una sola soluzione per un problema del tipo di Stefan a due fasi, unidimensionale, non lineare, relativo ad un mezzo termicamente isotropo, occupante uno strato piano indefinito di spessore finito. La non linearicà proveniva dall'aver supposto conduttività, densita, calore specifico di ciascuna fase funzioni della temperatura, soddisfacenti, unitamente allo stato termico iniziale e al flusso di calore assegnato in funzione del tempo su una delle due faccie dello strato, a condizioni del tutto aderenti al loro significato fisico.

E scopo di questo lavoro completare quella ricerca, come già feci nel caso lineare [2], esaminando l'analogo problema quando il mezzo termica. mente isotropo, interessato ad un cambiamento di fase, occupi uno strato cilindrico eircolare indefinito od uno strato sferico, nella ipotesi che lo stato termico nelle due fasi sia, ad ogni istante, funzione del posto soltanto tramite la distanza $r$ rispettivamente dall' asse delle due superficie cilindriche o dal centro comune alle due superficie sferiche limitanti lo strato.

Con questi due casi, la cui trattazione non è una banale estensione di quanto è stato già dimostrato per il caso piano, viene così completato, nelle nostre ipotesi, lo studio dei problemi nnidimensionali, non lineari, analoghi a quello di Stefan. Per una aggiornata bibliografia in argomento può vedersi [4].

(*) I numeri in parentesi [] si riferiscono alla bibliografia posta al termine del lavoro. 
2 - Posizione del problema ed equazione funzionale tra le incognite.

L'equazione parabolica della conduzione del calore che, nelle nostre ipotesi, con le notazioni di $(M)$, ̀े

$$
\rho(U) c(U) \frac{\partial U}{\partial t}=\operatorname{div}[K(U) \operatorname{grad} D]
$$

può scriversi in coordinate cilindriche:

$$
\frac{1}{r} \frac{\partial}{\partial r}\left[r K(U) \frac{\partial U}{\partial r}\right]=a(U) \frac{\partial U}{\partial t}
$$

$e$ in coordinate sferiche:

$$
\frac{1}{r^{2}} \frac{\partial}{\partial r}\left[r^{2} K(U) \frac{\partial U}{\partial r}\right]=a(U) \frac{\partial U}{\partial t}
$$

avendo in ogni caso posto $\rho(U) c(U)=a(U)$.

Queste due equazioni possono manifestamente riassumersi nell' unica equazione

$$
\frac{\partial}{\partial r}\left[r^{m-1} K(U) \frac{\partial U}{\partial r}\right]=r^{m-1} a(U) \frac{\partial U}{\partial r}, m=2,3 .
$$

Per $m=1$, ove si scriva $r=x$, si ritrova l'equazione del problema studiato in $(M)$.

Indicheremo con $0<R_{1}<R_{2}$ i raggi delle due superficie cilindriche o sferiche limitanti lo strato. Il problema, analogo a quello studiato in $(M)$, consiste nel considerare un mezzo termicamente isotropo, inizialmente costituito da una unica fase, nel quale $\grave{~}$ in atto un cambiamento di fase, originato all'istante $t=0+$ sulla superficie $r=R_{1}$ per effetto di una distri. buzione uniforme di sorgenti di rendimento specifico $H(t)$, creata sulla stessa superficie. Noto il calore latente $e$, quale funzione di $r$, lo stato termico iniziale $f(r)$ con $R_{1} \leq r \leq R_{2}$, supposta isolata la superficie $r=R_{2}$, nulla la temperatura critica e funzioni della temperatura le caratteristiche fisiche di ciascuna fase del mezzo, sono da determinare, ad ogni istante $t$ di un intervallo $(0, T)$, la temperatura in un punto qualsiasi delle due fasi e il raggio $r=\alpha(t)$ della superficie cilindrica o sferica, che all'istante $t$ separa le due fasi. Indicando con un indice 1 o 2 le grandezze relative 
alle due fasi, il nostro problema non lineare del tipo di Stefan, è analiti. camente ricondotto alla risoluzione dei due sistemi

$\left(A^{(1)}\right)$

$$
\begin{aligned}
& \frac{\partial}{\partial r}\left[r^{m-1} K_{1}\left(U^{(1)}\right) \frac{\partial U^{(1)}}{\partial r}\right]=r^{m-1} a_{1}\left(U^{(1)}\right) \frac{\partial U^{(1)}}{\partial \tau}, P \in D_{\alpha, t}^{(1)} ; \\
& {\left[K_{1}\left(U^{(1)}\right) \frac{\partial U^{(1)}}{\partial r}\right]_{r=R_{1}}=-H(\tau),} \\
& U^{(1)}[\alpha(t), t]=0, \alpha(0)=R_{1} \\
& D_{\alpha, t}^{(1)} \equiv\left\{0 \leq \tau \leq t ; R_{1}<r<\alpha(t)\right\} ; \\
& \frac{\partial}{\partial r}\left[r^{m-1} K_{2}\left(U^{(2)}\right) \frac{\partial U^{(2)}}{\partial r} \mid=r^{m-1} a_{2}\left(U^{(2)}\right) \frac{\partial U^{(2)}}{\partial \tau}, P \in D_{\alpha, t}^{(2)} ;\right. \\
& U^{(2)}(r, 0)=f(r), \\
& U^{(2)}[\alpha(t), t]=0, \alpha(0)=R_{1} \\
& {\left[\frac{\partial U^{(2)}}{\partial r}\right]_{r=R_{2}}=0,} \\
& D_{\alpha, t}^{(2)} \equiv\left\{0 \leq \tau \leq t ; \alpha(t)<r<R_{2}\right\}
\end{aligned}
$$

nélle incognite $U^{(1)}(r, \tau), U^{(2)}(r, \tau), \alpha(t)$ legate dalla relazione:

$$
\left[K_{2}\left(U^{(2)}\right) \frac{\partial U^{(2)}}{\partial r}-K_{1}\left(U^{(1)}\right) \frac{\partial U^{(1)}}{\partial r}\right]_{r=\alpha(t)}=M_{0} \dot{\alpha}(t)
$$

dove: $M_{0}$ è una costante positiva legata al calore latente; $K_{i}\left(U^{(i)}\right), a_{i}\left(U^{(i)}\right)$, $(i=1,2)$ sono funzioni positive limitate, per qualsiasi valore di $U^{(i)}$ nell' intervallo aperto $(-\infty,+\infty)$ ed ivi continue con le loro derivate prime, che supporremo limitate; $H(t)$ è una funzione positiva, continua con la sua derivata prima in $(O, T) ; f(r)$ ò una funzione continua con le due prime derivate in $\left(R_{1}, R_{2}\right)$ e tale che $f\left(R_{1}\right)=0, f^{\prime}(r) \leq 0, f^{\prime}\left(R_{2}\right)=0$, $f^{\prime \prime}(r) \geq 0$, gli apici indicando derivazione rispetto ad $r$ e $\dot{\alpha}=\frac{d \alpha}{d t}$. 
Con la trasformazione:

$$
V^{(i)}=V^{(i)}(r, \tau)=\int_{0}^{U^{(i)}} K_{i}(\xi) d(\xi)
$$

si ottiene da $\left(A^{(t)}\right)$ e $(B)$ :

$\left(\mathrm{I}^{(1)}\right)$

$$
\begin{aligned}
& \frac{\partial}{\partial r}\left(r^{m-1} \frac{\partial V^{(1)}}{\partial r}\right)=\frac{r^{m-2}}{k_{1}} \frac{\partial V^{(1)}}{\mathfrak{d} \tau}, P \in D_{\alpha, t}^{(1)} \\
& {\left[\frac{\partial V^{(1)}}{\partial r}\right]_{r=R_{1}}=-H(\tau)} \\
& V^{(1)}(\alpha, i)=0, \alpha(0)=R_{1}
\end{aligned}
$$

$$
\frac{\partial}{\partial r}\left(r^{m-1} \frac{\partial V^{(2)}}{\partial r}\right)=\frac{r^{m-1}}{k_{2}} \frac{\partial V^{(2)}}{\partial \tau}, P \in D_{\alpha, t}^{(2)}
$$$$
V^{(2)}(r, 0)=\psi(r),
$$

$\left(\mathrm{I}^{(2)}\right)$

$$
V^{(2)}(\alpha, t)=0, \alpha(0)=R_{1},
$$$$
\left[\frac{\partial V^{(2)}}{\partial r}\right]_{r=R_{2}}=0
$$

$$
\left[\frac{\partial V^{(2)}}{\partial r}-\frac{\partial V^{(1)}}{\partial r}\right]_{r=\alpha}=M_{0} \alpha
$$

dove :

$$
k_{i}=k_{i}\left(V^{(i)}\right)=\frac{K_{i}\left(V^{(i)}\right)}{a_{i}\left(V^{(i)}\right)} ; \quad \varphi(v)=\int_{0}^{f(r)} K_{2}(\xi) d \xi
$$

Queste mostrano per le ipotesi fatte su $K_{i}, a_{i}, f(r)$, che $k_{i}\left(^{*}\right)$ sono funzioni positive, continue e derivabili rispetto a $V^{(t)}$ e quindi almeno due volte rispetto ad $r$ ed una volta rispetto a $t$ e che $\varphi(r)$ soddisfa a

(*) Quando la cosa non possa dare luogo ad equivoco, tralasceremo la indicazione delle variabili da cui dipende una funzione. 
G. SEstun: Sul problema non Tineare di Stefan in strati cilindrici, ece. 197

tutte le ipotesi fatte su $f$, salvo al più quella relativa al segno della derivata seconda.

Con l'ulteriore posizione:

$$
\mathbf{Q}^{(i)}=\mathbf{Q}^{(v)}\left(V^{(i)}\right)=\int_{0}^{V^{(i)}} \frac{1}{k_{i}(\mu)} d \mu
$$

le prime due equazioni dei sistemi $\left(\mathrm{I}^{(i)}\right)$ si possono scrivere:

$$
\left(I^{(t)}, 1\right) \quad \frac{\partial}{\partial r}\left(r^{m-1} \frac{\partial V^{(i)}}{\partial r}\right)=r^{m-1} \frac{\partial Q^{(i)}}{\partial \tau}=\frac{\partial}{\partial \tau}\left(r^{m-1} \Omega^{(t)}\right) .
$$

La (4) mostra che le $\Omega^{(i)}$ sono funzioni continue e derivabili rispetto a $V^{(i)}$, e quindi rispetto ad $r$ e $t$, crescenti con $V^{(i)}$ ed aventi lo stesso segno di $V^{(i)}$.

Con procedimento analogo a quello seguito in $(M)$, integrando le $\left(I^{(i)}, 1\right)$ in $D_{a, t}^{(i)}$, si ottiene, tenuto conto di (II) e delle condizioni iniziale e al contorno,

$$
\alpha^{m}=A_{0}, m+F_{m}(l)-\frac{m}{M_{0}} \int_{R_{1}}^{\alpha} r^{m-1} \Omega^{(1)} d r-\frac{m}{M_{0}} \int_{\alpha}^{R_{2}} r^{m-1} \Omega^{(2)} d r
$$

dove

$$
A_{0}, m=\frac{m}{M_{0}} \int_{R_{1}}^{R_{2}} r^{m-1} \Omega^{(2)}[\varphi(r)] d r, \quad F_{m}(l)=R_{1}^{m}+\frac{m R_{1}^{m-1}}{M_{0}} \int_{0}^{t} H(\tau) d \tau .
$$

La (5) è la ricercata relazione funzionale che lega le incognite del problema e che cercheremo di risolvere con un algoritmo di approssimazioni successive.

3. - Qualche osservazione sulle soluzioni di sistemi quasi lineari del tipo $\left(I^{(i)}\right)$.

a) Scritte le equazioni $\left(I^{(i)}, 1\right)$ nella forma

$$
\frac{\partial^{2} V^{(i)}}{\partial r^{2}}+\frac{m-1}{r} \frac{\partial V^{(i)}}{\partial r}=\frac{1}{k_{\imath}\left(V^{(i)}\right)} \frac{\partial V^{(2)}}{\partial \tau}
$$

ò facile rendersi conto che continua a valere per soluzioni regolari di $\left(\mathrm{I}^{(i)}\right)$ un principio di massimo (P. M.) analogo a quello rilevato per le solu- 
zioni dei sistemi $\left(\mathrm{I}^{(i)}\right)$ di $(M)$. Quanto alle limitazioni per le soluzioni di sistemi analoghi a:

$\left(I^{\prime}\right)$

$$
\left\{\begin{array}{l}
\frac{\partial}{\partial r}\left(r^{m-1} \frac{\partial X}{\partial r}\right)=\frac{r^{m-1}}{k(X)} \frac{\partial X}{\partial \tau}, \quad P \in D_{\alpha, t}^{(1)} \\
{\left[\frac{\partial X}{\partial r}\right]_{r=R_{1}}=-H(\tau)} \\
X(\alpha, t)=0, \alpha(0)=R_{1},
\end{array}\right.
$$

ove $\alpha(t)$ si pensi funzione nota, crescente $e$ derivabile per ogni $t$ in $(0, T)$, converrà prima di tutto considerare l'integrale particolare $\psi_{m}(r, M)$ dell' equazione:

$$
\frac{d}{d r}\left(r^{m-1} \frac{d \psi}{d r}\right)=0
$$

soddisfacente alla condizione $\left(\frac{d \psi}{d r}\right)_{r=R 1}=-M$, con $M$ costante positiva.

Si ha manifestamente:

$$
\begin{array}{ll}
m=1 & \psi_{1}(r, M)=-M r \\
m=2 & \psi_{2}(r, M)=-M R_{1} \log \frac{r}{R_{1}} \\
m=3 & \psi_{3}(r, M)=-M R_{1}\left(1-\frac{R_{1}}{r}\right)
\end{array}
$$

e insieme che la funzione $W_{m}(r, M)=\psi_{m}(r, M)$ soddisfa al sistema:

$$
\left\{\begin{array}{l}
\frac{\partial}{\partial r}\left(r^{m-1} \frac{\partial W}{\partial r}\right)=\frac{r^{m-1}}{k(W)} \frac{\partial W}{\partial \tau}, P \in D_{\alpha, t}^{(1)} \\
{\left[\frac{\partial W}{d r}\right]_{r=R_{1}}=-M} \\
W(\alpha, t)=\psi_{m}(\alpha, M) .
\end{array}\right.
$$

Se allora assumiamo $M$ maggiore del massimo di $H(\tau)$ in $(0, t)$. la funzione $Z(r, t)=X(r, t)-W_{m}(r, M)$ soddisfa evidentemente il 
G. Sestini: Sul probtema non lineare di Stefan in strati cilindrici, ecc. 199

sistema :

$$
\left\{\begin{array}{l}
\frac{\partial}{\partial r}\left(r^{m-1} \frac{\partial Z}{\partial r}\right)=\frac{r^{m-1}}{k\left(Z+\psi_{m}\right)} \frac{\partial Z}{\partial \tau}, P \in D_{o, t}^{(1)} \\
\left(\frac{\partial Z}{\partial r}\right)_{r=R_{1}}=M-H(\tau)>0 \\
Z(\alpha, t)=-\psi_{m}(\alpha, M) \geq 0
\end{array}\right.
$$

Per il P. M. dovrà aversi $Z<-\psi_{m}(\alpha, M)$ e quindi per la $X$, soluzione di $\left(I^{\prime}\right)$ :

$$
X(r, \tau)=Z(r, \tau)+\psi_{m}(r, M)<\psi_{m}(r, M)-\psi_{m}(\alpha, M)
$$

e cioè, secondo i rari casi :

$$
\begin{array}{ll}
m=1 & X<M(\alpha-r) \\
\mathrm{m}=2 & X<M R_{1}(\log \alpha-\log r)
\end{array}
$$

$$
m=3 \quad X<M R_{\tilde{1}}^{2} \frac{\alpha-r}{\alpha r}
$$

con $M$ indipendente da $\alpha$. La prima delle (9) coincide, come doveva essere, con la $(6)$ di $(M)$.

Giova per il seguito osservare che in $D_{x, t}^{(1)} \dot{e} X(r, \tau) \geq 0$. Infatti deve essere $X\left(R_{1}, \tau\right) \geq 0$ perchè, essendo $\left(\frac{\partial X}{\partial r}\right)_{r=R_{1}}<0$ e $X(\alpha, t)=0$, in caso contrario la $X$ avrebbe internamente a $D_{x, t}^{(1)}$ un minimo negativo, contrariamente a quanto prescrive il P. M.; essendo allora la $X \geq 0$ sul contorno del campo, sempre per P. M., tale dovrà essere anche internamente a $D_{\alpha, t}^{(1)}{ }^{*}$ Osserveremo che, essendo $\left(\frac{\partial X}{\partial r}\right)_{r=R_{1}}<0, X(\alpha, t)=0$ e $X(r, \tau) \geq 0$ in $D_{\alpha, t}^{(1)}$, deve essere, per $r=\alpha, \frac{\partial X}{\partial r} \leq 0$. Ciò porta che anche internamente a $D_{\alpha, t}^{(1)}$ deve essere $\frac{\partial X}{\partial r} \leq 0$. Infatti se ciò non avvenisse, per la continuità di $\frac{\partial X}{\partial r}$, per ogni $\bar{t}$ in $(0, t)$ si avrebbero due valori di $r$ per i quali $\frac{\partial X}{\partial r}$ si annullerebbe cambiando segno. Per uno di questi valori la $X(r, \bar{t})$ avrebbe un massimo relativo positivo. Questo però non è possibile. Infatti con la 
sostituzione $X=x e^{M \tau}$, con $M$ costante positiva, operata sulla equazione $\left(I^{\prime}, 1\right)$ si avrebbe

$$
\frac{\partial^{2} x}{\partial r}+\frac{m-1}{r} \frac{\partial x}{\partial r}=\frac{M x}{k}+\frac{1}{k} \frac{\partial x}{\partial \tau}
$$

che non potrebbe restare verificata nel punto di massimo positivo di $X(r, \bar{l})$, che è pure massimo positivo per $x(r, \bar{t})$, in quanto, essendo internamente a $D_{x, t}^{(1)}$ limitata la $\frac{\partial X}{\partial \tau}$ si può, scegliendo $M$ convenientemente grande, rendere positivo il secondo membro della precedente equazione.

b) Consideriamo ora il sistema:

(II')

$$
\left\{\begin{array}{l}
\frac{\partial}{\partial r}\left(r^{m-1} \frac{\partial Y}{\partial r}\right)=\frac{r^{m-1}}{k(Y)} \frac{\partial Y}{\partial \tau}, P \in D_{\alpha, t}^{(2)} \\
Y(r, 0)=\varphi(r) \\
Y(\alpha, t)=0 \\
{\left[\frac{\partial Y}{\partial r}\right]_{r=R_{2}}=0}
\end{array}\right.
$$

e nel campo $\bar{D}_{x, t}^{(2)}$, ottenuto da $D_{\alpha, t}^{(2)}$ per simmetria rispetto alla retta $r=R_{2}$ il sistema:

$$
\left\{\begin{array}{l}
\frac{\partial}{\partial r}\left(r^{m-1} \frac{\overline{\partial y}}{\partial r}\right)=\frac{r^{m-1}}{k(\bar{y})} \frac{\partial \bar{y}}{\partial \tau}, P \in \bar{D}_{\jmath_{,}}^{(2)} \\
\bar{y}(\alpha, t)=\bar{y}\left(2 R_{2}-\alpha, t\right)=0 \\
\bar{y}(r, 0)=\bar{\varphi}(r)
\end{array}\right.
$$

$\operatorname{con}$

$$
\bar{\varphi}(r)=\varphi(r), \text { per } \quad r \leq R_{2} ; \bar{\varphi}(r)=\varphi\left(2 R_{2}-r\right), \text { per } r \geq R_{2}
$$

La soluzione $\bar{y}$ che, nelle ipotesi ammesse, esiste regolare ed unica [1], per essere negativa o nulla sul contorno tale risulta per P. M. anche nell'interno di $\bar{D}_{x, t}^{(2)}$ ed essendo nel sottocampo $D_{\alpha, t}^{(2)} \bar{y}(r, \tau)=Y(r, \tau)$, si ha che la soluzione regolare di (II') è negativa o nulla in $D_{\alpha, t}^{(2)}$. 
G. Sestini: Sul problena non lineare di Stefan un strati cilindrici, cec. 201

Operiamo ora in $\left(\mathrm{II}^{\prime}\right)$ la sostituzione:

$$
0 \leq-Y(r, \tau)=W(r, \tau)-\varphi(r)+M \tau
$$

con $M$ costante positiva, ottenendo:

$$
\left\{\begin{array}{l}
\left.\frac{\partial}{\partial r} \mid r^{m-1}\left(\frac{\partial W}{\partial r}-\varphi^{\prime}\right)\right]=\frac{r^{m-1}}{k(W, r, \tau)}\left(\frac{\partial W}{\partial \tau}+M\right), P \in D_{\alpha, t}^{(2)} \\
W(r, 0)=0 \\
W(x, t)=\varphi(\alpha)-M t \\
{\left[\frac{\partial W}{\partial r}\right]_{r=R_{2}}=0 .}
\end{array}\right.
$$

Se allora consideriamo in $\bar{D}_{\alpha, t}^{(2)}$ il sistema:

$$
\left\{\begin{array}{l}
\frac{\partial}{\partial r}\left(r^{m-1} \frac{\partial Z}{\partial v}\right)=\frac{r^{m-1}}{k(Z, r, \tau)} \frac{\partial Z}{\partial \tau}+r^{m-1}\left(\frac{M}{k(Z, r, \tau)}+\frac{m-1}{r} \varphi^{\prime}+\psi^{\prime \prime}\right), P \in \bar{D}_{\alpha, t}^{(2),} \\
Z(r, 0)=0, \\
Z(\alpha, t)=Z\left(2 R_{2}-\alpha, t\right)=\varphi(\alpha)-M t,
\end{array}\right.
$$

valendo per esso un teorema di esistenza ed unicità di soluzioni regolarí [1], si ha $W(r, \tau)=Z(r, \tau)$ in $D_{\alpha, t}^{(2)}$. Se allora scegliamo la costante positiva $M$ in modo che sia $\frac{M}{k(Z, r, \tau)}+\frac{m-1}{r} \varphi^{\prime}+\varphi^{\prime \prime}>0$, il che $\dot{e}$ possibile nelle nostre ipotesi, la $Z$ risulta in tutto $D_{x, t}^{(2)}$ non superiore al massimo dei valori assunti sul contorno e percio la $W$ in $D_{\alpha, t}^{(2)}$, essendo la $\varphi(r)$ decrescente, verificherà la disuguaglianza

$$
W(r, \tau) \leq \varphi(\alpha)-M t
$$

da cui

$$
0 \leq-Y(r, \tau) \leq \varphi(\alpha)-\varphi(r)
$$

c) Siano ora $X_{\alpha}$ ed $X_{\beta}$ due soluzioni regolari di $\left(I^{\prime}\right)$, relative a due campi $D_{\alpha, t}^{(1)}$ e $D_{\beta, t}^{(1)}$ rispettivamente, con $\alpha>\beta$ funzioni note, crescenti e 
derivabili di $t$, con $\alpha(0)=\beta(0)=R_{1}$. In $D_{\beta, t}^{(1)}$ si ha evidentemente, posto

$$
\begin{gathered}
\varepsilon_{\alpha \beta}=X_{\alpha}-X_{\beta}, \\
\frac{\partial}{\partial r}\left(r^{m-1} \frac{\partial \varepsilon_{\alpha \beta}}{\partial r}\right)=\frac{r^{m-1}}{k\left(X_{\beta}\right)} \frac{\partial \varepsilon_{\alpha \beta}}{\partial \tau}+\Phi\left(\varepsilon_{\alpha \beta}, r, \tau\right), P \in D_{\beta, t}^{(1)} ; \\
{\left[\frac{\partial \varepsilon_{a \beta}}{\partial r}\right]_{r=R_{1}}=0,} \\
\left(\varepsilon_{\alpha \beta}\right)_{r=\beta}=X_{a}(\beta, t) \geq 0,
\end{gathered}
$$

con

$$
\Phi=\Phi\left(\varepsilon_{\alpha \beta \beta}, r, \tau\right)=r^{m-1}\left[\frac{1}{k\left(X_{\beta}+\varepsilon_{\alpha \beta \beta}\right)}-\frac{1}{k\left(X_{\beta}\right)}\right] \frac{\partial X_{\alpha}}{\partial \tau},
$$

che, per le ipotesi ammesse, risulta limitata per qualsiasi valore di $\varepsilon_{\alpha \beta}, r$ e $\tau$. Con la sostituzione $\varepsilon_{\alpha \beta}=W_{\alpha \beta} e^{M \tau}$, con $M$ costante positiva, il precedente sistema si trasforma in:

$$
\left\{\begin{array}{l}
\frac{\partial}{\partial r}\left(r^{m-3} \frac{\partial W_{\alpha \beta}}{\partial r}\right)=\frac{r^{m-1}}{k\left(X_{\beta}\right)} \frac{\partial W_{\alpha \beta}}{\partial \tau}+\frac{r^{m-1} M}{k\left(X_{\beta}\right)} W_{\alpha \beta}+e^{-M \tau} \Phi\left(W_{\alpha \beta}, r, \tau\right), P \in D_{\beta, t}^{(1)} \\
{\left[\frac{\partial W_{\alpha \beta}}{\partial r}\right]_{r=R_{1}}=0} \\
\left(W_{\alpha \beta}\right)_{r=\beta}=e^{-M t} X_{\alpha}(\beta, t) \geq 0 .
\end{array}\right.
$$

Essendo $|\Phi| \leq N, \frac{r^{m-1}}{k}>0$, si può scegliere $M$ così grande che

$$
\bar{\Phi}\left(W_{\alpha \beta}, r, \tau\right)=\frac{r^{m-1}}{k} M W_{\alpha \beta}+e^{-M \tau} \Phi\left(W_{\alpha \beta}, r, \tau\right)
$$

abbia il segno di $W_{\alpha \beta}$ e quindi il segno di $\varepsilon_{\alpha \beta}$. $W_{\alpha \beta}$ (e quindi $\varepsilon_{\alpha \beta}$ ) non può percio avere massimi positivi o minimi negativi in $D_{\beta, t}^{(1)}$ e quindi, se positiva o nulla sul contorno di $D_{\beta, t}^{(1)}$ (se negativa o nulla), tale dovrà risultare anche internamente. Essendo $[a)] X_{\alpha}(r, \tau) \geq 0$ qualunque siano $r$ in $\left(R_{1}, \beta\right)$ e $\tau$ in $(0, t)$, sard̀ provato che $W_{\alpha \beta} \geq 0$ in $D_{\beta, t}^{(1)}$ non appena sarà dimostrato che è $W_{\alpha \beta} \geq 0$ per $r=R_{1}$. Ragionando per assurdo sia $W_{\alpha \beta}<0$ per $r=R_{1}$, a 
G. Sestixi: Sul problema non lineare di Stefan in strati cilindrici, ecc. 203

partire da un certo istante $t^{*}$. Per $t \leq t^{* *}$ sufficientemente piccolo, nel senso che risulti $\beta(t) \leq R_{1}-\delta$ con $\delta>0$, è senz'altro $W_{\alpha \beta}(r, \tau) \geq 0$, come sarebbe facile dimostrare col metodo gia usato in $(M)$ in quanto il campo $\bar{D}_{B, t}^{(1)}$, ottenuto da $D_{\beta, t}^{(1)}$ per simmetria rispetto alla retta $r=R_{1}$, non contenendo la retta $r=0$, consentirebbe di trarre conclusioni analoghe a quelle cui si giungeva in $(M)$. Per $\bar{t} \geq t^{*}$ in $(0, t)$ si ha allora $W_{\alpha \mathrm{p}}\left(\bar{t}, R_{1}\right)<0$ ed insieme $W_{x \beta}[\beta(\bar{t}), \bar{t}]=X_{x}[\beta(\bar{t}), \bar{t}] e^{-M \bar{t}} \geq 0$. Sulla caratteristica $\tau=\bar{t}$, dovrà esistere un $r^{*}$, compreso tra $R_{1}$ e $\beta(\bar{t})$, per cui è $W_{\alpha, \beta}\left(r^{*}, \bar{t}\right)=0$ e, passando $W_{\alpha \beta}$ da valori negativi a valori positivi, si avrà: $\left[\frac{\partial W_{\alpha \beta}}{\partial r}\right]_{r=r^{*}, \tau=t} \geq 0$. Avendosi poi $[a)]\left[\frac{\partial X_{\alpha}}{\partial r}\right]_{r=\beta, \tau=\bar{t}} \leq 0$ e quindi, per la continuità sia di $\frac{\partial W_{\alpha \beta}}{\partial r}$ che di $\frac{\partial X_{\alpha}}{\partial r}$, anche $\left[\frac{\partial W_{\alpha \beta}}{\partial r}\right]_{r=\beta, \tau=\bar{t}} \leq 0$, ci sarà internamente all' intervallo $\left[r^{*}, \beta(\bar{t})\right]$ un punto $r^{\prime}$ in cui $\frac{\partial W_{\alpha \beta}(r, \bar{t})}{\partial r}$ si annulla, passando da valori positivi a valori negativi. La $W_{\alpha e}(r, \bar{t})$ ha perciò per $r=r^{\prime}$ un massimo relativo positivo. Ciò è però impossibile in quanto, essendo internamente a $D_{\beta, t}^{(1)} \frac{\partial W_{\alpha \beta}}{\partial \tau}$ limitata, sce. gliendo $M$ sufficientemente grande. non potrebbe restare verificata nel punto $r^{\prime}, \bar{t}$ la $[(11), 1]$. Essendo allora per ogni $\bar{t}$ di $(0, t) \quad W_{a p}\left(R_{1}, \bar{t}\right) \geq 0$, segue, per $P . M$., $W_{\alpha \beta}(r, \tau) \geq 0$, e quindi $\varepsilon_{\alpha \beta} \geq 0$, in $D_{\beta, t}^{(1)}$.

Ricordando poi la (8) si ha:

$$
0 \leq \varepsilon_{\alpha \beta} \leq \max X_{\alpha}(\beta, t)<\psi_{m}(\beta, M)-\psi_{m}(\alpha, M),
$$

eon $\psi_{m}(r, M)$ data dalle (9).

d) Considerando infine due solazioni regolari $Y_{\alpha} \otimes Y_{\beta}$ di (II') rispettivamente in $D_{\alpha, t}^{(2)}$ e $D_{\beta, t}^{(2)}$ con $\alpha>\beta$. In $D_{\alpha, t}^{(2)}$, posto $\delta_{\alpha \beta}=Y_{\alpha}-Y_{\beta}$, si ha:

$$
\left\{\begin{array}{l}
\frac{\partial}{\partial r}\left(r^{m-1} \frac{\partial \delta_{\alpha, \beta}}{\partial r}\right)=\frac{r^{m-1}}{k\left(\delta_{\alpha \beta}+Y_{\beta}\right)} \frac{\partial \delta_{\alpha \beta}}{\partial \tau}+\psi\left(\delta_{\alpha \beta}, r, \tau\right), \quad P \in D_{\alpha, t}^{(2)} \\
\delta_{\alpha \beta}(r, 0)=0 \\
{\left[\frac{\partial \delta_{\alpha \beta}}{\partial r}\right]_{r=R_{2}}} \\
\delta_{\alpha \beta}(\alpha, t)=-Y_{\beta}(\alpha, t) \geq 0
\end{array}\right.
$$

con

$$
\psi=\psi\left(\delta_{\alpha \beta}, r, \tau\right)=r^{m-1}\left[\frac{1}{k\left(\delta_{\alpha \beta}+Y_{\beta}\right)}-\frac{1}{k\left(Y_{\beta}\right)}\right] \frac{\partial Y_{\beta}}{\partial \tau} .
$$


204 G. Sestini: Sul problema non lineare di Stefan in strati cilindrici, ecc.

Ragionando su questo sistema come sull' analogo (14) di $(M)$, introdn. cendo un sistema ausiliario nel campo ottenuto da $D_{\alpha, t}^{(2)}$ per simmetria intorno alla retta $r=R_{2}$, si ottiene $\delta_{\alpha \beta} \geq 0$ in $D_{\alpha, t}^{(2)}$.

Ricordando la (10), si ha poi:

$$
0 \leq \delta_{x \beta} \leq \max \left[-Y_{\beta}(x, t)\right] \leq \varphi(\beta)-\varphi(x)
$$

4. - Proprietà delle successive approssimazioni per $\alpha(t)$.

Per la risoluzione di (5) istituiamo un algoritmo di approssimazioni successive ponendo:

$$
\left\{\begin{array}{l}
\alpha_{0}^{m}=F_{m}(t), \\
\alpha_{n}^{m}=A_{0}, m+F_{m}(t)-\frac{m}{M_{0}} \int_{R_{1}}^{\alpha_{n-1}} r^{m-1} \Omega_{n-1}^{(1)} d r-\frac{m}{M_{0}} \int_{\alpha_{n-1}}^{R_{2}} r^{m-1} \Omega_{n-1}^{(2)} d r
\end{array}\right.
$$

dove $\Omega_{n-1}^{(i)}$ sta per $\boldsymbol{\Omega}^{(i)}\left(V_{n-1}^{(i)}\right)$ e $V_{n-1}^{(i)}$ sono soluzioni regolari dei sistemi ottenuti da (I (i) e (II) facendovi $\alpha_{n-1}$ al posto di $\alpha$.

Le $\alpha_{n}$ sono funzioni monotone crescenti di $t$ e complessivamente limitate. Infatti da

$$
\begin{gathered}
\alpha_{n}^{m b}=\alpha_{0}^{m}+\frac{m}{M_{0}} \int_{R_{1}}^{\alpha_{n-1}} r^{m-1} \Omega^{(2)}[\varphi(r)] d r+ \\
+\frac{m}{M_{0}} \int_{\alpha_{n-1}}^{R_{2}} r^{m-1}\left[\Omega^{(2)}(\varphi)-\Omega^{(2)}\left(V_{n-1}^{(2)}\right)\right] d r-\frac{m}{M_{0}} \int_{R_{1}}^{\alpha_{n-1}} r^{m-1} \Omega_{n-1}^{(1)} d r,
\end{gathered}
$$

essendo $\Omega_{n-1}^{(1)} \geq 0$ perchè (N. $\left.3, a\right) V_{n-1}^{(1)} \geq 0, \frac{m}{M_{0}} \int_{B_{1}}^{\alpha_{n-1}} r^{m-1} \Omega^{(2)}(\varphi) d r<0$ e $\mathbf{Q}^{(2)}(\varphi)-$ - $\mathrm{Q}^{(2)}\left(V_{n-1}^{(2)}\right) \leq 0$, per essere $\Omega^{(2)}$ crescente e $V_{n-1}^{(2)} \geq \varphi(r)$, non potendo avere $V_{n-1}^{(2)} \leq 0$ minimi negativi in $D_{\alpha_{n-1}}^{(2)}, t \operatorname{segue}: \alpha_{n}^{m} \leq \alpha_{0}^{m}$ e, per essere $\alpha_{n}$ ed $\alpha_{0}$ positive:

$$
0<\alpha_{n}(t) \leq \alpha_{0}(t)
$$

Gioveră qui osservare che il processo di cambiamento di fase, dovendosi avere, per la (16) e la prima delle $(15), \alpha_{n}^{m}(t) \leq \alpha_{0}^{m}(t)=F_{m}^{\prime}(t)$, qualunque sia $n$, durerà fin tanto che $F_{m}(t) \leq R_{2}^{m}$. Si ha perciò un estremo superiore 
per $t$ imponendo

$$
F_{m}(t)=R_{1}^{n}+\frac{m R_{1}^{m-1}}{M_{0}} \int_{0}^{t} H(\tau) d \tau \leq R_{2}^{m}
$$

Nell'ipotesi di $(M)$ che $H(t)$ sia crescente, e tutte le volte in eui è $\lim _{t \rightarrow \infty} F_{m}(t)>R_{2}^{m}$, ciò che certamente accade se per qualunque $t$ in $(0,+\infty)$ si ha $H(t) \geq H_{0}>0$, con $H_{0}$ costante, la (17) stabilisce effettivamente, come la (17) di $(M)$, una limitazione per $t$. Se però la $H(t)$, continua e positiva, è tale che $\lim _{t \rightarrow \infty} H(t)=0$, può accadere che sia $\lim _{t \rightarrow \infty} F_{m}(t) \leq R_{2}^{m}$ perciò la (17), essendo la $F_{n}(l)$ funzione crescente di $t$, resta soddisfatta per ogni $t \geq 0$ e non pone alcnna limitazione per il tempo.

Che in generale ci sia una limitazione per la durata del fenomeno e che su tale durata, e quindi sulla velocità di avanzamento $\dot{\alpha}$, abbia influenza determinante il flusso $H(t)$, creato sulla parete $r=R_{1}$, ̀̀ del resto fisicamente intuitivo.

Derivando la seconda delle (15) rispetto a $t$, si ha:

$$
\begin{gathered}
m x_{n}^{m-1} \dot{\alpha}_{n}=\frac{m R_{1}^{m-1}}{m_{0}} H(t)-\frac{m}{M_{0}} \int_{R_{1}}^{\alpha_{n-1}} r^{m-1} \frac{\partial Q^{(1)}}{\partial t} d r-\frac{m}{M_{0}} \int_{\alpha_{n-1}}^{R_{2}} r^{m-1} \frac{\partial Q^{(2)}}{\partial t} d r= \\
=\frac{m R_{1}^{m-1}}{M_{0}} H(t)-\frac{m}{M_{0}} \int_{R_{1}}^{\alpha_{n-1}} \frac{\partial}{\partial r}\left[r^{m-1} \frac{\partial V_{n-1}^{(1)}}{\partial r}\right] d r-\frac{m}{M_{0}} \int_{\alpha_{n-1}}^{R_{2}} \frac{\partial}{\partial r}\left[r^{m-1} \frac{\partial V_{n-1}^{(2)}}{\partial r}\right] d r= \\
=\frac{m}{M_{0}} \alpha_{n-1}^{m-1}\left[\frac{\partial V_{n-1}^{(2)}}{\partial r}-\frac{\partial V_{n-1}^{(1)}}{\partial r}\right]_{r=\alpha_{n-1}} .
\end{gathered}
$$

$\grave{E}$ facile rendersi conto che è:

$$
\alpha_{n}=\frac{1}{M_{0}}\left(\frac{\alpha_{n-1}}{\alpha_{n}}\right)^{m-1}\left[\frac{\partial V_{n-1}^{(2)}}{\partial r}-\frac{\partial V_{n-1}^{(1)}}{\partial r}\right]_{r=\alpha_{n-1}}>0 .
$$

Infatti sappiamo $(\mathrm{N} .3, a)$ che $\lim _{r \rightarrow a_{n-1}} \frac{\partial V_{n-1}^{(1)}}{\partial r} \leq 0$. Essendo poi $(N .3, b)$ $V_{n-1}^{(2)}(r, t) \leq 0$ ed avendosi $V_{n-1}^{(2)}\left(\alpha_{n-1}, t\right)=0$, dovrà aversi $\underset{r \rightarrow \alpha_{n}+1}{\lim } \frac{\partial \nabla_{n-1}^{(2)}}{\partial r} \geq 0$. 
D'altra parte i due limiti non possono essere entrambi nguali a zero in quanto vi sarebbe con ciò continuità nel flusso di calore attraverso $r=\alpha_{n-1}$, contrariamente all'ipotesi che attraverso alla superficie di separazione tra le due fasi vi sia assorbimento (o emissione) di calore.

Segue perciò, come volevamo, la (17).

\section{5. - Teorema di esistenza ed unicità.}

Procedendo in modo analogo a quanto fatto nei NN. 8 e 9 di $(M)$, valendo per le $\alpha_{n}, \nabla_{n}^{(1)}$ e $V_{n}^{(2)}$ proprietà e limitazioni analoghe, si può agevolmente dimostrare l'esistenza di una sola soluzione per il problema espresso dai sistemi $\left(\mathrm{I}^{(i)}\right)$ con la (II), restando anche valido il teorema relativo alle approssimazioni $\alpha_{n}$ di $\alpha$, esposto al N. 10 di $(M)$, che afferma: due approssimazioni successive per $\alpha(t)$, secondo le (15), soddisfano alla disuguaglianza opposta a quella che intercede tra la coppia di approssima. zioni di indici immediatamente precedenti, restando in conseguenza assicurato sia un criterio di scelta per estrarre da $\left\{\alpha_{n}\right\}$ una sottosuccessione conver. gente $\left\{\overline{\alpha_{n}}\right\}$, sia la possibilità di approssimare per eccesso e per difetto l'incognita $\alpha(t)$, alla cui determinazione è stato in sostanza ricondotto il problema considerato.

Ci limiteremo qui a ricordare le linee generali del teorema di esistenza

Essendo la $\left\{\alpha_{n}\right\}$ una successione di funzioni positive, complessivamente limitate, si potrà estrarre da essa una sottosuccessione $\left\{\bar{\alpha}_{n}\right\}$ convergente ad un limite determinato e finito $\alpha^{*} \leq \alpha_{0}$. Se $\overline{\nabla^{(i)}}$ sono le soluzioni regolari dei sistemi ottenati da $\left({ }^{(i)}\right)$ e (II) facendovi $\alpha=\alpha^{*}$ e si scrive

$$
\alpha^{\prime m}=A_{0},{ }_{m}+F_{m}(t)-\frac{m}{M_{0}} \int_{0}^{\alpha^{*}} r^{m-1}{\overline{\Omega^{(1)}}}^{(1)} d r-\frac{m}{M_{0}} \int_{\alpha^{*}}^{R_{2}} r^{m-1}{\overline{\mathbf{Q}^{(2)}}}^{(2)}
$$

dove si è posto $\overline{\boldsymbol{\Omega}}^{(i)}=\mathbf{\Omega}^{(i)}\left(\bar{\nabla}^{(i)}\right)$, si ha $\alpha^{\prime m}=\alpha^{* m}$ e quindi $\alpha^{\prime}=\alpha^{*}$.

Infatti, supposto ad es. $\alpha^{*} \geq \bar{\alpha}_{n-1}$, da

$$
\begin{gathered}
\alpha^{\prime m}-\bar{\alpha}_{n}^{m}=\frac{m}{M_{0}} \int_{0}^{\bar{\alpha}_{n-1}} r^{m-1}\left(\bar{\Omega}_{n-1}^{(1)}-\bar{\Omega}^{(1)}\right) d r-\frac{m}{M_{0}} \int_{\bar{\alpha}_{n-1}}^{\alpha^{*}} r^{m-1}{\overline{Q^{(1)}}}^{(1)} d r+ \\
+\frac{m}{M_{0}} \int_{\alpha^{*}}^{R_{2}} r^{m-1}\left(\overline{\mathbf{Q}}_{n-1}^{(2)}-\bar{\Omega}^{(2)}\right) d r-\frac{m}{M_{0}} \int_{\alpha^{*}}^{\bar{\alpha}_{n-1}} r^{m-1} \bar{\Omega}_{n-1}^{(2)} d r,
\end{gathered}
$$


si ha, ricordando le proprietà di $\Omega^{(i)}$ e le $(10),(12)$ :

$$
\begin{gathered}
\left|\alpha^{\prime m}-\bar{\alpha}_{n}^{m}\right| \leq \frac{m}{M_{0}} \int_{0}^{\alpha_{n-1}} r^{m-1}\left|\bar{\Omega}_{n-1}^{(1)}-\Omega^{(1)}\right| d r+\frac{2 m M}{M_{0}}\left(\alpha^{*}\right)^{m-1} \mid \alpha^{*}-\left(\bar{\alpha}^{n-1} \mid+\right. \\
\quad+\frac{m}{M_{0}} \int_{\alpha^{*}}^{R_{z}} r^{m-1}\left|\overline{\mathbf{Q}}_{n-1}^{(2)}-\overline{\mathbf{Q}}^{(2)}\right| d r \leq \\
\leq \frac{R_{2}^{m}}{M_{0}} N\left\{\left|\psi_{m}\left(\alpha^{*}, M\right)-\psi_{m}\left(\bar{\alpha}_{n-1}, M\right)\right|+\left|\varphi\left(\bar{\alpha}_{n-1}\right)-\varphi\left(\alpha^{*}\right)\right|+\right. \\
\left.\quad+\frac{2 m M}{N}\left|\alpha^{*}-\bar{\alpha}_{n-1}\right|\right\}
\end{gathered}
$$

dove $N$ è il più grande dei massimi di $\frac{1}{k_{i}\left(\nabla^{(i)}\right)}, i=1,2$.

Ricordando le espressioni (7) per $\psi_{m}$ e le ipotesi su $\varphi(r)$, segue che, scelto un $\sigma>0$ arbitrario, è possibile determinare un $n_{0}$ tale che per $n-1>n_{0}$, sia :

$$
\left|\alpha^{\prime m}-\alpha_{n}^{m}\right|<\sigma
$$

e da ciò la coincidenza di $\alpha^{\prime}$ con $\alpha^{*}$. Il completamento del teorema si ottiene con la dimostrazione della derivabilità di $\alpha(t)$, come in $(M)$, con metodo diretto.

La dimostrazione del teorema di unicità si consegue come al N. 9 di $(M)$.

\section{BIBLIOGRAFIA}

[1] M. Grvaex, Equations aux dérivées partielles du type parabolique, «J. Math. Pures A ppl. », (6), 9, 306-471, 1013.

[2] G. Sestrur, Esistenza ed unicita nel problema di Stefan relativo a campi dotati di simmetria, «Rivista Mat. Univ. Parma ", 3, 103-113, 1952.

[3] - -, Sul problema unidimensionale non lineare di Stefan in uno strato piano indefinito, *Ann. Mat. Pura Appl.»(4), 51, 203-22t, 1960.

[4] - , Problemi di diffusione lineari e non lineari analoghi a quello di Stefan, Conferenze Sem. Mat. Univ. Bari, N.i 55-56, 1-26, 1960. 